PROCEEDINGS OF THE

AMERICAN MATHEMATICAL SOCIETY

Volume 129, Number 10, Pages 3025-3029

S 0002-9939(01)06062-2

Article electronically published on April 17, 2001

\title{
FINITE TIME BLOW UP FOR A NAVIER-STOKES LIKE EQUATION
}

\author{
STEPHEN MONTGOMERY-SMITH
}

(Communicated by David S. Tartakoff)

\begin{abstract}
We consider an equation similar to the Navier-Stokes equation. We show that there is initial data that exists in every Triebel-Lizorkin or Besov space (and hence in every Lebesgue and Sobolev space), such that after a finite time, the solution is in no Triebel-Lizorkin or Besov space (and hence in no Lebesgue or Sobolev space). The purpose is to show the limitations of the so-called semigroup method for the Navier-Stokes equation. We also consider the possibility of existence of solutions with initial data in the Besov space $\dot{B}_{\infty}^{-1, \infty}$. We give initial data in this space for which there is no reasonable solution for the Navier-Stokes like equation.
\end{abstract}

In this paper, we consider a simplified model for the Navier-Stokes equation what we call the cheap Navier-Stokes equation. For this equation, we show that for sufficiently large initial data, we get blow up in finite time. The purpose of this is not to indicate the possibility that the Navier-Stokes equation might blow up in finite time - indeed the author strongly believes the opposite. Rather, the purpose of this paper is to show limitations in some of the methods used in studying the Navier-Stokes equation.

Let us consider the following version of the Navier-Stokes equation:

$$
\frac{\partial u}{\partial t}=\Delta u-P(\operatorname{div}(u \otimes u)),
$$

where $t \mapsto u_{t}$ is an $\mathbb{R}^{n}$ valued function, or tempered distribution, on $\mathbb{R}^{n}$. Here $P$ denotes the Leray projection that takes a vector field to its divergence free part. A tremendous amount of work has been done on the difficult problem of determining if the solutions exist, if they are unique, and to which spaces they belong. One approach, which we consider in this paper, is to consider mild solutions using what is often called the semigroup approach. This is described in [Ca1], and is used in many papers, for example, [FK], [K], GM], [KT]. An example of this kind of result is that due to Kato [K], who showed that if $u_{0} \in L_{n}\left(\mathbb{R}^{n}\right)^{n}$, then there is a solution $u \in C\left([0, T],\left(L_{n}\left(\mathbb{R}^{n}\right)\right)^{n}\right)$ if either $T=T\left(u_{0}\right)$ is sufficiently small and $\left\|u_{0}\right\|_{L_{n}}$ is arbitrary or $\left\|u_{0}\right\|_{L_{n}}$ is small and $T=\infty$.

Received by the editors March 1, 2000.

2000 Mathematics Subject Classification. Primary 35Q30, 46E35; Secondary 34G20, 37L05, 47D06, 47H10.

Key words and phrases. Navier-Stokes equation, semigroup, fixed point method, TriebelLizorkin space, Besov space.

The author was partially supported by NSF grant DMS 9870026 .

(C)2001 American Mathematical Society 
However, if one studies all these papers, one sees that they do not use all of the properties of the Navier-Stokes equation. Indeed, the methods seem to apply equally well to the following cheap Navier-Stokes equation:

$$
\frac{\partial u}{\partial t}=\Delta u+\sqrt{-\Delta}\left(u^{2}\right)
$$

Here $t \mapsto u_{t}$ is a scalar valued function or tempered distribution on $\mathbb{R}^{n}$. The semigroup approach is to consider a mild solution of the cheap Navier-Stokes equation, that is, a solution to the equation $u=G(u)$, where $G: C([0, T], X) \rightarrow C([0, T], X)$, with $X$ being a space of tempered distributions on $\mathbb{R}^{n}$ :

$$
\left.G(u)_{t}=e^{t \Delta} u_{0}+\int_{0}^{t} e^{(t-s) \Delta} \sqrt{-\Delta}\left(u_{s}^{2}\right)\right) d s .
$$

The method used to find a fixed point of $G$ is to show that $G$ is a contraction mapping on $C([0, T], X)$ or on some subset of $C([0, T], X)$. (Often one cannot obtain the result by working directly with $C([0, T], X)$. For example, in the case $X=L_{n}\left(\mathbb{R}^{n}\right)$ one has to work with some space embedded into $X$; as is shown by Oru [O], the map $G$ is not even continuous on $C\left([0, T], L_{n}\left(\mathbb{R}^{n}\right)\right)$.)

The semigroup method by itself never seems to be able to obtain global results, that is, results valid for both $T$ and the size of $u_{0}$ arbitrarily large. To obtain global results, one has to appeal to other kinds of estimates for the Navier-Stokes equation, for example, energy estimates (see, for example, $\mathrm{KF}$. or [Ca2]).

The purpose of this paper is to show that the semigroup method really is limited in this way. If we could obtain global results for the Navier-Stokes equation using only the semigroup method, then the same methods would also apply to the cheap Navier-Stokes equation. This would then contradict the main result of this paper and its corollary.

Thus, if one is going to obtain global results for the Navier-Stokes equation, one has to consider properties of the bilinear form $P(\operatorname{div}(u \otimes u))$ that are not shared by the bilinear form $\sqrt{-\Delta}\left(u^{2}\right)$. Perhaps there is some mysterious cancellation property in the first bilinear form that causes global regularity for the Navier-Stokes equation. In any case, the semigroup technique itself, at least in the manner in which it has been applied to date, is not going to solve the problem.

Let $e_{1}$ denote the first unit vector in $\mathbb{R}^{n}$, and for $x \in \mathbb{R}^{n}, r>0$, let $B_{r}(x)$ denote $\{y:|x-y| \leq r\}$. Let $\hat{u}$ denote the Fourier transform of $u$ with respect to $x \in \mathbb{R}^{n}$.

Theorem 1. Let $w$ be a tempered Schwartz function such that $\hat{w}$ is non-negative, has $L_{1}$ norm equal to 2 , and has support in $B_{1 / 4}\left(3 e_{1} / 4\right) \cup B_{1 / 4}\left(-3 e_{1} / 4\right)$. (So $w$ is in every Triebel-Lizorkin or Besov space.) Then if $A>2^{13 / 3}$, and if $u$ is a mild solution to the cheap Navier-Stokes equation whose Fourier transform is nonnegative, with initial data $u_{0}=A w$, then $u_{t}$ is not in any Triebel-Lizorkin or Besov space when $t=\log \left(2^{1 / 3}\right)$.

Corollary 2. Let $w$ be as in Theorem 1 (so $w \in L_{n}\left(\mathbb{R}^{n}\right)$ ), and let $A>2^{13 / 3}$. Then there is no mild solution $u$ to the cheap Navier-Stokes equation, with $u_{0}=A w$, in $C\left([0, T], L_{n}\left(\mathbb{R}^{n}\right)\right)$, for $T=\log \left(2^{1 / 3}\right)$.

We also present another result about the cheap Navier-Stokes equation. For the Navier-Stokes equation (and hence also for the cheap Navier-Stokes equation), it turns out that the natural spaces in which to consider solutions are of the form $C([0, T], X)$, where $X$ is scale invariant, that is, $\|\lambda f(\lambda \cdot)\|_{X}=\|f\|_{X}$ for all $\lambda \in$ 
$(0, \infty)$. Authors have considered which is the largest scale invariant space for which one gets existence results. For example, recently Koch and Tataru [KT] showed such results when $X$ is the space of derivatives of functions with bounded mean oscillation.

In fact, it can be shown (arguing similarly as in Frazier, Jawerth and Weiss in FJW for the minimality of $B_{1}^{0,1}$ ) that all scale invariant spaces of distributions, that also contain all Schwartz functions, are contained in the Besov space $\dot{B}_{\infty}^{-1, \infty}$. Cannone Ca1 was able to obtain results for the Navier-Stokes equation in the space $\dot{B}_{p}^{n / p-1, \infty}, n \leq p<\infty$, but left open the case $p=\infty$ corresponding to the space $\dot{B}_{\infty}^{-1, \infty}$.

We will respond to this last case negatively for the cheap Navier-Stokes equation. Although this does not answer the question for the Navier-Stokes equation, the author believes that it is very possible that there is a similar non-existence result for the Navier-Stokes equation. But this would say more about the nature of the space $\dot{B}_{\infty}^{-1, \infty}$ than about the Navier-Stokes equation itself.

Theorem 3. Let $w$ be a tempered Schwartz function whose Fourier transform is non-negative, and has support in $B_{1}(0) \backslash B_{1 / 2}(0)$. Let $v$ be the tempered distribution

$$
v(x)=\sum_{k=1}^{\infty} 2^{k} \cos \left(\left(2^{k}-1\right) x_{1}\right) w(x) .
$$

Then $v \in \dot{B}_{\infty}^{-1, \infty}$, but there is no mild solution $u \in C\left([0, T], \mathcal{S}^{\prime}\right)$ to the cheap Navier-Stokes equation, with non-negative Fourier transform, and with $u_{0}=v$, for any $T>0$. (Here $\mathcal{S}^{\prime}$ denotes the space of tempered distributions.)

The crucial observation for all these results is that

$$
\widehat{G(u)}_{t}(\xi)=e^{-t|\xi|^{2}} \hat{u}_{0}+\int_{0}^{t} e^{(s-t)|\xi|^{2}}|\xi|\left(\hat{u}_{s} * \hat{u}_{s}\right)(\xi) d s
$$

(here $*$ denotes convolution). Thus we see that if $\hat{u}, \hat{v} \geq 0$, and that $\hat{u} \leq \hat{v}$, then $\widehat{G(u)} \leq \widehat{G(v)}$.

Proof of Theorem 11. Using the usual embedding theorems, it may be seen that all of the Triebel-Lizorkin and Besov spaces embed into $\dot{B}_{\infty}^{a, \infty}$ for some $a \in \mathbb{R}$. Let us recall the definition of the Besov space $\dot{B}_{\infty}^{a, \infty}$. Let $\phi(x)$ be some tempered Schwartz function whose Fourier transform is non-negative, whose support is "mostly" contained in a band about $|\xi|=1$, and such that $\sum_{k=-\infty}^{\infty} \hat{\phi}_{k}$ is uniformly bounded above and below. Here $\phi_{k}(x)=2^{n k} \phi\left(2^{k} x\right)$. Then $\dot{B}_{\infty}^{a, \infty}$ is the space of distributions on $\mathbb{R}^{n}$ for which the norm $\|f\|_{\dot{B}_{\infty}^{a, \infty}}=\sup _{k} 2^{a k}\left\|\phi_{k} * f\right\|_{L_{\infty}}$ is finite.

Write $w_{0}$ for the function $\hat{w}_{0}=\hat{w} I_{B_{1 / 4}\left(3 e_{1} / 4\right)}$, and set $w_{k}=w_{0}^{2^{k}}$. Since $w$ and $\hat{w}$ are real valued, we see that $\hat{w}$ must be an even function. Hence we quickly see that $\hat{w}_{k}$ has $L_{1}$ norm equal to 1 , and is supported in $\left\{\xi: 2^{k-1} \leq|\xi| \leq 2^{k}\right\}$.

We will show by induction that $\hat{u}_{t} \geq A^{2^{k}} \alpha_{k}(t) \hat{w}_{k}$ for $k>0$, where $\alpha_{k}(t)=$ $2^{k-4\left(2^{k}-1\right)} e^{-2^{k} t} I_{t \geq t_{k}}, t_{0}=0$, and $t_{k}=\log (2) \sum_{j=1}^{k} 2^{-2 j}$. The case $k=0$ follows since $\hat{u}_{t}(\xi) \geq e^{-t|\xi|^{2}} \hat{u}_{0}(\xi)$. Suppose that our desired inequality holds for $k-1$. 
Then

$$
\begin{aligned}
\hat{u}_{t}(\xi) & \left.\geq \int_{0}^{t} e^{(s-t)|\xi|^{2}}|\xi|\left(\hat{u}_{s} * \hat{u}_{s}\right)(\xi)\right) d s \\
& \geq \int_{0}^{t} e^{2^{2 k}(s-t)} 2^{k-1}\left(A^{2^{k-1}} \alpha_{k-1}(s)\right)^{2}\left(\hat{w}_{k-1} * \hat{w}_{k-1}\right)(\xi) d s \\
& \geq A^{2^{k}} 2^{k-4\left(2^{k}-1\right)} 2^{1+2 k} \int_{t_{k-1}}^{t} e^{2^{2 k}(s-t)} e^{-2^{k} s} d s \hat{w}_{k}(\xi) \\
& \geq A^{2^{k}} 2^{k-4\left(2^{k}-1\right)} e^{-2^{k} t} 2^{1+2 k} \int_{t_{k-1}}^{t} e^{2^{2 k}(s-t)} d s \hat{w}_{k}(\xi) \\
& =A^{2^{k}} 2^{k-4\left(2^{k}-1\right)} e^{-2^{k} t} 2\left(1-e^{2^{2 k}\left(t_{k-1}-t\right)}\right) \hat{w}_{k}(\xi) \\
& \geq A^{2^{k}} 2^{k-4\left(2^{k}-1\right)} e^{-2^{k} t} \hat{w}_{k}(\xi)
\end{aligned}
$$

whenever $1-e^{2^{2 k}\left(t_{k-1}-t\right)} \geq 1 / 2$, that is, $t_{k}-t_{k-1} \geq 2^{-2 k} \log (2)$.

Let $t_{\infty}=\lim _{k \rightarrow \infty} t_{k}=\log \left(2^{1 / 3}\right)$. Since $\hat{\phi}_{k} \hat{u}_{t_{\infty}} \geq A^{2^{k}} \alpha_{k}\left(t_{\infty}\right) \hat{w}_{k} \geq 0$,

$$
\begin{aligned}
\left\|u_{t_{\infty}}\right\|_{\dot{B}_{\infty}^{a, \infty}} & =\sup _{k} 2^{a k}\left\|\phi_{k} * u_{t_{\infty}}\right\|_{L_{\infty}} \\
& =\sup _{k} 2^{a k}\left\|\hat{\phi}_{k} \hat{u}_{t_{\infty}}\right\|_{L_{1}} \\
& \geq \sup _{k} A^{2^{k}} 2^{a k+k-4\left(2^{k}-1\right)} e^{-2^{k} t_{\infty}} .
\end{aligned}
$$

It is clear that this is infinite if $A>2^{4} e^{t_{\infty}}=2^{13 / 3}$.

Proof of Corollary 2 Suppose for a contradiction that there is a solution $u \in$ $C\left([0, T], L_{n}\left(\mathbb{R}^{n}\right)\right)$. By the methods of $[\mathrm{K}]$, we know that there is a number $\epsilon>0$, depending only upon $\|u\|$, such that for every $t \in[0, T]$ there is a mild solution $v \in C\left([t, t+\epsilon], L_{n}\left(\mathbb{R}^{n}\right)\right)$ to the cheap Navier-Stokes equation, with $v_{t}=u_{t}$, that is obtained by starting with $v_{s}^{(0)}=e^{(s-t) \Delta} u_{t}$, and iterating a function $G$ similar to that defined above. By the uniqueness result of Furioli, Lemarié-Rieusset and Terraneo [FLT], we have that $v_{s}=u_{s}$ for $t \leq s \leq t+\epsilon$. From this it is clear that if $\hat{u}_{t} \geq 0$, then $\hat{u}_{s} \geq 0$ for $t \leq s \leq t+\epsilon$. Applying this argument several times, we see that $\hat{u}_{t} \geq 0$ for $0 \leq t \leq T$. Then by Theorem 1, $u_{T}$ is not in any Triebel-Lizorkin space, and hence in particular is not in $L_{n}\left(\mathbb{R}^{n}\right)$.

Proof of Theorem [3. Suppose we have a non-negative solution $u:[0, T] \rightarrow \mathcal{S}^{\prime}$ to the cheap Navier-Stokes equation with $u_{0}=v$. It is clear that

$$
\begin{aligned}
\hat{u}_{t}(\xi) & \geq e^{-t|\xi|^{2}} \hat{v}(\xi) \\
& \geq \sum_{k=1}^{\infty} 2^{k} e^{-2^{2 k}} \frac{1}{2}\left(\hat{w}\left(\xi+\left(2^{k}-1\right) e_{1}\right)+\hat{w}\left(\xi-\left(2^{k}-1\right) e_{1}\right)\right) .
\end{aligned}
$$


Thus

$$
\begin{aligned}
\hat{u}_{t}(\xi) & \left.\geq \int_{0}^{t} e^{(s-t)|\xi|^{2}}|\xi| \hat{(} u_{s} * \hat{u}_{s}\right)(\xi) d s \\
& \geq \int_{0}^{t} \sum_{k=1}^{\infty} e^{4(s-t)} 2^{2 k-1} e^{-2^{2 k+1} s}(\hat{w} * \hat{w})(\xi) d s \\
& =\left(\sum_{k=1}^{\infty} 2^{2 k-1} e^{-4 t} \int_{0}^{t} e^{\left(4-2^{2 k+1}\right) s} d s\right)(\hat{w} * \hat{w})(\xi) \\
& =\left(\sum_{k=1}^{\infty} \frac{2^{2 k-1}}{2^{2 k+1}-4} e^{-4 t}\left(1-e^{\left(4-2^{2 k+1}\right) t}\right)\right)(\hat{w} * \hat{w})(\xi),
\end{aligned}
$$

which is infinite on the support of $\hat{w} * \hat{w}$ for $t>0$, and hence $u_{t}$ cannot be in $\mathcal{S}^{\prime}$.

The author would like to thank Marco Cannone for many useful discussions and helpful remarks.

\section{REFERENCES}

[Ca1] Cannone, Marco, Ondelettes, paraproduits et Navier-Stokes. (French) [Wavelets, paraproducts and Navier-Stokes] With a preface by Yves Meyer. Diderot Editeur, Paris, 1995. MR 2000e:35173]

[Ca2] Cannone, Marco, Rôle des oscillations et des espaces de Besov dans la résolution des équations de Navier-Stokes. Document de synthèse présenté pour obtenir une Habilitation à diriger des Recherches, Université de Paris 7, 1-35, 1999.

[FJW] Frazier, Michael; Jawerth, Björn; Weiss, Guido, Littlewood-Paley theory and the study of function spaces. CBMS Regional Conference Series in Mathematics, 79. Published for the Conference Board of the Mathematical Sciences, Washington, DC; by the American Mathematical Society, Providence, RI, 1991. MR 92m:42021

[FK] Fujita, Hiroshi; Kato, Tosio, On the Navier-Stokes initial value problem. I. Arch. Rational Mech. Anal. 161964 269-315. MR 29:3774

[FLT] Furioli, Giulia; Lemarié-Rieusset, Pierre-Gilles; Terraneo, Elide, Sur l'unicité dans $L^{3}\left(\mathbb{R}^{3}\right)$ des solutions "mild" des équations de Navier-Stokes. [On the uniqueness in $L^{3}\left(\mathbb{R}^{3}\right)$ of mild solutions for the Navier-Stokes equations] C. R. Acad. Sci. Paris Sér. I Math. 325 (1997), no. 12, 1253-1256. MR 98g:35159

[GM] Giga, Yoshikazu; Miyakawa, Tetsuro, Navier-Stokes flow in $\mathbb{R}^{3}$ with measures as initial vorticity and Morrey spaces. Comm. Partial Differential Equations 14 (1989), no. 5, 577-618. MR 90e:35130

[K] Kato, Tosio, Strong $L^{p}$-solutions of the Navier-Stokes equation in $\mathbb{R}^{m}$, with applications to weak solutions. Math. Z. 187 (1984), no. 4, 471-480. MR 86b:35171

[KF] Kato, Tosio; Fujita, Hiroshi On the non-stationary Navier-Stokes system, Rend. Sem. Mat. Univ. Padova 32:243-260, 1962. MR 26:495

$[\mathrm{KT}]$ Koch, Herbert; Tataru, Daniel, Well-posedness for the Navier-Stokes equation, preprint.

[O] Oru F., Rôle des oscillations dans quelques problèmes d'analyse non linéaire. Thèse de Doctorat de l'ENS Cachan, 1998.

Department of Mathematics, University of Missouri, Columbia, Missouri 65211

E-mail address: stephen@math.missouri.edu

$U R L:$ http://www.math.missouri.edu/ ${ }^{\text {stephen }}$ 